\title{
Polarization Insensitive Fiber-Optic Refractometer based on a Side-polished Fiber Coupler with a Thick Glass Waveguide
}

\author{
Kyung-Rak Sohn* and Jae-Won Song \\ School of Electrical Engineering and Computer Science, Kyungpook National University, \\ Daegu 702-701, KOREA \\ Hyung-Pyo Kim \\ School of Electronic and Electrical Engineering, Sangju National University, \\ Kyungpook 742-711, KOREA
}

(Received July 8, 2002)

\begin{abstract}
Side-polished single-mode fiber covered with a thick planar-glass-waveguide is studied as a polarization-insensitive fiber-optic refractometer. The properties of the device are very sensitive to the index variation of an upper cladding on top of the overlay waveguide. We investigate the spectral response, the polarization properties, and the relationship between the shift in coupling wavelength and the index variation of an upper cladding layer. Index resolutions of the order of 10-4 have been achieved.

OCIS codes : $060.1810,060.2370,230.5440,230.7390$.
\end{abstract}

\section{INTRODUCTION}

Side-polished fiber couplers covered with a planar multi-mode waveguide are one of the most useful methods to utilize the optical fiber sensors. These devices exhibit periodic power transfer from a fiber to an overlay waveguide as a function of input wavelength and the transverse mode order of the overlay waveguide. The transmission responses therefore depend on the material properties of an overlay waveguide and its upper cladding layers. Many applications based on these couplers have been proposed and investigated as temperature $[1,2]$ pressure [3], humidity [4], and refractive index sensors $[5,6]$. Usually the overlay waveguides are fabricated by the spin coating of polymers, polishing of a $\mathrm{LiNbO}_{3}$ [7] or BK7 glass, and evaporation of the dielectric materials [8]. But polymers and dielectric layers are very weak for physical and (or) chemical attacks. The $\mathrm{LiNbO}_{3}$ and BK7 glass are polished up to desired thickness (i.e. several or several tens micrometer). Furthermore, these sensors that utilize such multimode overlay waveguides always suffer from the polarization sensitivity due to the asymmetric overlay waveguide structure $[2,5,6]$. Polarization insensitivity of in-line fiber sensors is very important in practical devices. If the external pertur- bations such as temperature, pressure, humidity, and refractive index are measured as a function of transmitted optical intensity, the sensors require polarization independence characteristics to ensure the reliability, easy fabrication process, low cost, and simple structure.

In this work, we present a polarization-insensitive in-line fiber refractometer using side-polished fiber couplers. Despite the asymmetric overlay waveguide structure, we have experimentally proved that the coupling wavelengths for TE and TM polarizations nearly coincide. This is due to the polarization dependence being reduced when the thickness of an overlay waveguide increases [9]. Herein, the commercial microscope cover glass with thickness of a $170 \mu \mathrm{m}$ is used in role of the overlay waveguide. We have also shown that these devices are capable of measuring the refractive index as a function of the optical power through the refractometer.

\section{PRINCIPLE OF OPERATION}

The basic operating principle of the side-polished fiber coupler is evanescent field coupling between a side-polished single-mode fiber and an overlay waveguide. Strong power transfer from the fiber to the over 


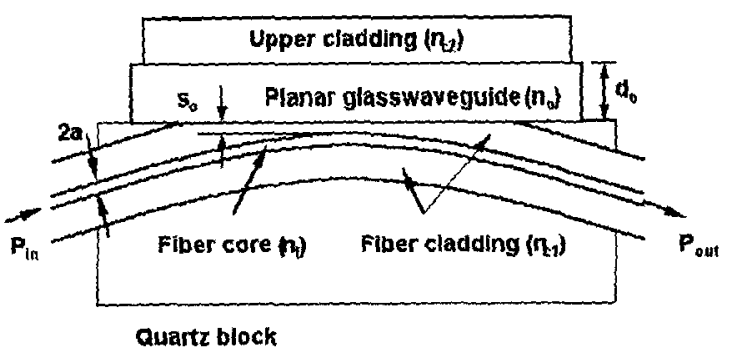

FIG. 1. Schematic of a proposed side-polished fiber refractometer

lay waveguide occurs only if the effective index of two waveguides is matched $\left(n_{e f}=n_{o f}\right)$. The proposed sensor based on this coupler is composed of a sidepolished single mode fiber and a planar glass waveguide as shown in Fig. 1. Herein, we denote that the refractive index of a fiber core, a fiber cladding, an overlay waveguide, and an upper cladding as $n_{f}, n_{\mathrm{cl}}$, $n_{o}$, and $n_{c 2}$, respectively. The thickness of an overlay waveguide and a remaining cladding of the fiber are $d_{0}$ and $s_{0}$, respectively. A qualitative analysis of this structure can be explained by observing the modal properties of a fiber and an overlay. Since the glass overlay can be considered to be the planar waveguide and these modes are highly dispersive, the wave length response shows the periodic filter (i.e. comb filter) behavior [7]. The coupling wavelengths are approximately given by the following equation derived from the transverse resonance conditions of an overlay waveguide $[8]$.

$$
\lambda_{\nu}=\frac{2 \pi d_{0}\left(n_{\mathrm{o}}^{2}-n_{\mathrm{ef}}^{2}\right)^{1 / 2}}{\nu \pi+\phi_{c 1}+\phi_{\mathrm{c} 2}}
$$

where $\lambda_{\nu}$ is the input wavelength. $n_{o}$ is the index of an overlay waveguide and $n_{e f}$ is the effective index of a fiber core. $\nu$ is the transverse mode order. $\phi_{c 1}$ and $\phi c 2$, given by the following equation, are the phase shifts occurred at the lower and upper boundaries of an overlay waveguide, respectively.

$$
\phi_{c i}=\tan ^{-1} \zeta \frac{\left(n_{e o}^{2}-n_{c i}^{2}\right)^{1 / 2}}{\left(n_{\mathrm{o}}^{2}-n_{e f}^{2}\right)^{1 / 2}}, \quad i=1,2
$$

where $\zeta$ is the polarization-dependent constant which is equal to one for TE modes and $\left(n_{o} / n_{c i}\right)^{2}$ for TM modes.

If the thickness of an upper cladding layer in these refractometers is greater than the penetration depth of the evanescent field of an overlay waveguide, the upper cladding index seen by the modes of the overlay waveguide appears as a bulk medium. So, we can obtain the wavelength shift $(\Delta \lambda)$ for a change in upper cladding index by manipulation of the eigen-

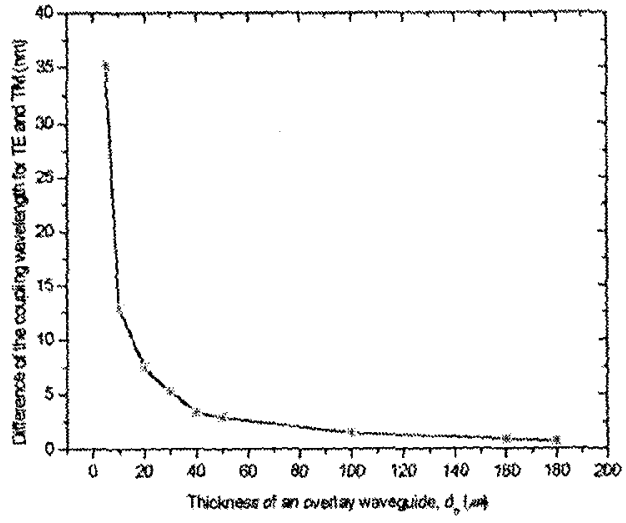

FIG. 2. Difference of a coupling wavelength for a TE and a TM polarization as a function of the thickness of a planar glass waveguide.(calculated)

value equation of (1). If the coupling wavelength in the air cladding $\left(n_{c 2}=1\right)$ is $\lambda_{0}$ and that in the changed cladding index $\left(n_{c 2}=n_{c 2}^{\prime}\right)$ is $\lambda^{\prime}$, then the shift in coupling wavelength, $\Delta \lambda=\lambda^{\prime}-\lambda_{0}$, is given by

$$
\Delta \lambda=\frac{\lambda_{0}^{2}\left(\phi_{c 2}-\phi_{\mathrm{c} 2}^{\prime}\right)}{2 \pi d_{o}\left(n_{\mathrm{o}}^{2}-n_{e f}^{2}\right)^{1 / 2}-\lambda_{0}\left(\phi_{c 2}-\phi_{c 2}^{\prime}\right)}
$$

where $\phi_{c 2}$ and $\phi_{\mathrm{c} 2}^{\prime}$ are given by (2) with air $\left(n_{c 2}=1\right)$ and changed index $\left(n_{\mathrm{c} 2}=n_{\mathrm{c} 2}^{\prime}\right)$, respectively. From (3), the coupling wavelength is thus sensitive to the index change in the upper cladding via phase shift $\phi_{c 2}^{\prime}$

But, if the overlay waveguide is asymmetric (i.e. $\left.n_{c 1} \neq n_{c 2}\right)$, the coupling wavelength for two orthogonal polarizations is different. The overlay waveguide having the different boundary conditions for TE and TM polarizations shows the structural birefringence so that the effect of phase shift $\phi_{i}$ cannot be neglected.

To reduce this effect, we can control the thickness of an overlay waveguide. The difference of a coupling wavelength for TE and TM polarizations using (3) is plotted in terms of a thickness of a glass overlay in Fig. 2. When the thickness of an overlay waveguide increases, that difference is reduced exponentially.

\section{EXPERIMENTAL RESULTS}

To obtain the partially polished single-mode fiber, the fiber is fixed into the convex-grooved quartz block to support the macro-bend shape and cemented by an epoxy resin. After that the quartz block is carefully polished up to a distance $\left(s_{0}\right)$ of $1.5 \mu \mathrm{m}$ to the fiber core to get access to the evanescent field of the guiding mode in the fiber. The distance is calculated by using the radiated coupling loss due to the high index liquid 


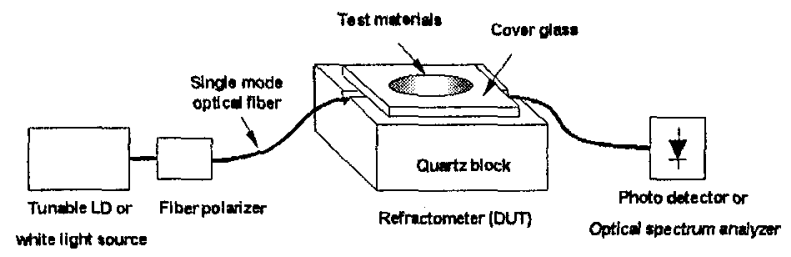

FIG. 3. Experimental setup of a side-polished fiber refractometer.

drop on top of the polished fiber [9]. And then the commercial microscope-cover-glass with thickness of a $170 \mu \mathrm{m}$ is bonded on the side-polished fiber block. This cover glass is adequate for our device. No additional polishing procedure was required to achieve the thickness desired as an overlay waveguide, and it is not susceptible to chemical and physical attacks. The material birefringence is also ignored.

Fig. 3 shows the experimental set-up. For obtaining the transmission spectrum of the fabricated device, the white light source is installed at the end of the input fiber. A fiber polarizer is inserted between the input source and the input fiber of the device to verify the polarization properties due to the structural birefringence of an overlay waveguide. The fundamental wavelength-response with air shows comb filtering characteristics with the free spectral range of a $19 \mathrm{~nm}$, the coupling depth of above $7 \mathrm{~dB}$, and the insertion loss of below $1.5 \mathrm{~dB}$. To investigate the shift in transmission spectrum according to index variation of an upper cladding, the Cargille refractive-index oils with known refractive index in range 1.3 to 1.448 are used in the role of the upper cladding. Fig. 4 shows the spectral response for TE and TM input polarizations

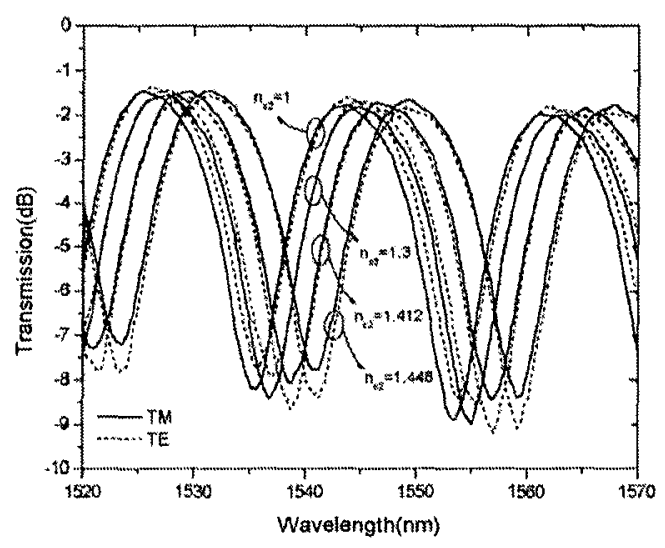

FIG. 4. Measured spectral responses for a TE and a TM polarization as a function of an upper cladding index.

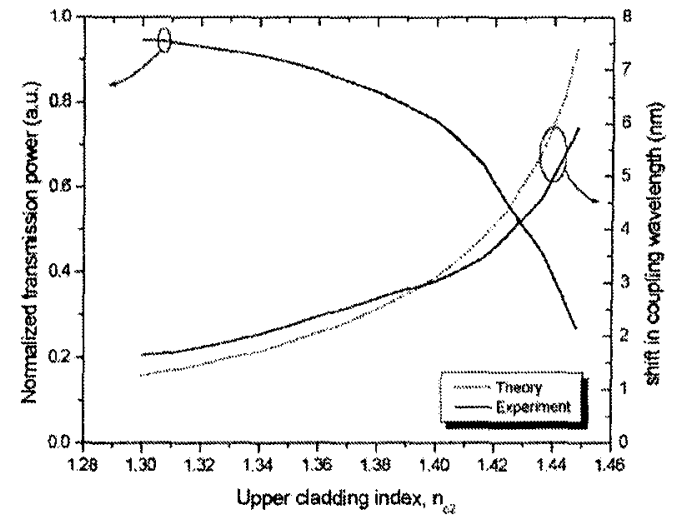

FIG. 5. Measured relative transmission power and shift in coupling wavelength as a function of an upper cladding index. The center wavelength and launching power of an input light source are a $1543 \mathrm{~nm}$ and $1 \mathrm{~mW}$, respectively.

in terms of the upper cladding index. Two coupling depths in the given wavelength range correspond to the coupling wavelength. In our experiment, the optical spectra are shifted to longer wavelength by increasing the upper layer index. It shows clearly that the coupling wavelength is strongly dependent on the upper cladding index. Furthermore, the coupling wavelengths are nearly in agreement with each other for two orthogonal polarizations. The measured polarization dependence loss (PDL) is less than $0.3 \mathrm{~dB}$. We have experimentally proven that the polarizationinsensitivity of the side-polished fiber coupler is easily realized by the thick overlay such as a microscope cover glass.

These polarization-insensitive characteristics lead to a miniaturized in-line fiber refractometer. For the previously reported ones $[5,6]$, the shift range in coupling wavelength and the polarization dependence are very large and hence it's not adequate to represent the index variation in terms of a transmitted optical power at specified wavelength. One is only identified as the shift in coupling wavelength using an optical spectrum analyzer. In our results, since the maximum wavelength shift in given upper cladding index range is about $6 \mathrm{~nm}$ and less than half of a periodic as shown in Fig. 4, we can investigate the optical power variation corresponding to the shift in coupling wavelength at specified wavelength. A tunable erbium-doped fiber (EDF) ring laser is used as a light source. The center wavelength of the tunable laser is adjusted to 1543 $\mathrm{nm}$ and optical power of $1 \mathrm{~mW}$ is launched into the device. The lightwave multimeter is used to measure the transmitted optical power corresponding to the refractive index of the liquid materials dropped on top 
of the cover glass. Fig. 5 depicts its relative relationship. While the transmission spectra shift to longer wavelength by increasing an upper layer index, the optical output power is reduced in proportion to that. These results clearly indicate the possibility of identifying the index variation as transmitted intensity. For a change of output power of $1 \mu \mathrm{W}$, the performance of the experimental device shows that the minimum detectable change in upper cladding index of $6.4 \times 10^{-4}$ is feasible.

\section{CONCLUSION}

We have demonstrated the polarization-insensitive in-line fiber refractometer using a side-polished fiber coupler covered with a microscope cover glass with thickness of a $170 \mu \mathrm{m}$. It is based on that the coupler is very sensitive to the index variation of an upper cladding on top of the overlay waveguide. The index resolution of the fabricated refractometer in terms of the transmitted power is $6.4 \times 10^{-4} / \mu \mathrm{W}$. As a thick overlay waveguide is adopting, the polarization sensitive caused by the structural birefringence of an overlay waveguide is also minimized. The PDL is less than $0.3 \mathrm{~dB}$. It is believed that the proposed device may be applied to achieve the polarizationindependence and miniaturized fiber-optic sensors.

*Corresponding author : hyryu@mail.kaist.ac.kr.

\section{REFERENCES}

[1] A. Alvarez-Herrero, H. Guerrero, T. Belenguer, and D. Levy, IEEE Photon. Technol. Lett. 12, 1043 (2000).

[2] W. G. Jung, S. W. Kim, K. T. Kim, E. S. Kim, and S. W. Kang, IEEE Photon. Technol. Lett. 13, 1209 (2001).

[3] S. Lee, S. Kim, H. Rue, J. Lee, and J. Song, OSA annual meeting, (Optical Society of America, Rhode Island, USA, 2000), paper TuG5.

[4] F. P. Corera, A. Gaston, and J. Sevilla, Proc. IMTC, (IEEE, Baltimore, USA, 2000), 17.

[5] W. Johnstone, G. Fawcett, and L. W. K. Yim, IEE Proc.-Optoelectron, 141, 299 (1994).

[6] G. Raizada and B. P. Pal, Opt. Lett. 21, 399 (1996).

[7] K. R. Sohn and J. W. Song, Opt. Commun. 203, 271 (2002).

[8] D. G. Moodie and W. Johnstone, Opt. Lett. 12, 1025 (1993).

[9] K. T. Kim, H. W. Kwon, J. W. Song, S. Lee, W. G. Jung, and S. W. Kang, Opt. Commun. 180, 37 (2000). 